\title{
Uso de ropivacaína a $0,5 \%$ em anestesia peribulbar de gatos
}

\section{Use of $\mathbf{0 . 5 \%}$ ropivacaine for peribulbar anesthesia in cats}

\author{
Francisco Cláudio Dantas Mota, ${ }^{*}$ Duvaldo Eurides, ${ }^{* *}$ Patricia Maria Coletto Freitas, ${ }^{* * *}$ Marco Antonio de Andrade Belo, ${ }^{*}$ \\ Vando Edésio Soares, ${ }^{*}$ Luana Alexandra Fatoretto****
}

\begin{abstract}
Resumo
Objetivou-se verificar a eficiência da ropivacaína na anestesia peribulbar em 10 gatos adultos sem raça definida, machos e fêmeas com peso variando de 2 a $3 \mathrm{~kg}$. Os animais foram anestesiados com clorpromazina (1,0 mg/kg VO) e propofol (3,0 $\mathrm{mg} / \mathrm{kg} \mathrm{IV).} \mathrm{Foi} \mathrm{introduzida} \mathrm{uma} \mathrm{agulha} \mathrm{hipodérmica} \mathrm{de} 13$ × 3,8 mm em forma de "L" na porção dorsal da cavidade orbitária em direção ao fórnice, e administrado $1,0 \mathrm{mg} / \mathrm{kg}$ de ropivacaína a 0,5\%. Decorridos 10 minutos e um segundo observou-se proptose com duração de 100 minutos e seis segundos e perda do reflexo corneano após 10 minutos e cinco segundos, em média, com permanência de 124 minutos e dois segundos. O globo ocular manteve-se concêntrico por 121 minutos e cinco segundos, sem nistagmo. A ropivacaína a $0,5 \%$ no bloqueio peribulbar em gatos, ocasiona centralização do globo ocular e abole os reflexos corneanos.
\end{abstract}

Palavras-chave: gatos, ropivacaína, peribulbar, anestesia.

\begin{abstract}
The peribulbar anesthesia is largely used in corrective surgeries, such as glaucoma, cataract, and keratoplasty. The purpose of this study was to verify the efficacy of $0.5 \%$ ropivacaine for peribulbar anesthesia in cats. Ten adult cats, male and female, weighing between 2 and $3 \mathrm{~kg}$, were used. The animals received chlorpromazine (1,0 mg/kg, orally) and propofol (3,0 mg/kg, intravenously). Ropivacaine at $0.5 \%(1,0 \mathrm{mg} / \mathrm{kg})$ was administered by using a L-shape hypodermic needle (13x3.8mm), which was introduced in the upper portion of the orbital cavity towards the fornix. The onset of proptosis was observed in $10.1 \mathrm{~min}$ and lasted $100.6 \mathrm{~min}$. The loss of the corneal reflex occurred after 10.5min and lasted $124.2 \mathrm{~min}$. The ocular globe maintained concentric for $121.5 \mathrm{~min}$ and did not present nistagmus. It can be concluded that $0.5 \%$ ropivacaine solution induces centralization of the ocular globe, abolishes the corneal reflexes without causing any collateral effects in cats.
\end{abstract}

Keywords: cats, ropivacaine, peribulbar, anesthesia.

\section{Introdução}

Os bloqueios retrobulbares e peribulbares têm sido empregados com frequência nas cirurgias oftálmicas em humanos (Cangiani et al.,1995). Na medicina veterinária, esta prática é limitada nos procedimentos mais invasivos e de longa duração (Chaves et al., 1997). Nos últimos anos, surgiram novas técnicas cirúrgicas oftálmicas e agentes anestésicos que proporcionam conforto e segurança para o paciente e cirurgião (Bechara, 2002).

A anestesia peribulbar favorece as cirurgias corretivas de catarata, glaucoma e outras intervenções oftálmicas (Chaves et al. 1997), obedecendo aos princípios gerais da cirurgia ocular, por promover imobilidade absoluta, baixa pressão intraocular, diminuição do sangramento e abolição do reflexo oculocardíaco (Cangiani et al., 1995).

As complicações relacionadas com a anestesia retrobulbar são lesões no nervo óptico, hemorragia retrobulbar, quemo- se, depósitos subconjuntivais (Gelatt, 1991), intensa dor ocular, parada cardiorrespiratória (Freitas e Espirandelli, 1997) e apnéia (Ramakrishna, 1995). Já anestesia peribulbar apresenta menores complicações em relação à anestesia retrobulbar, o que aumenta sua indicação nas cirúrgias oculares (Morgan et al., 1988). Entretanto, já foram relatados perfuração do globo ocular e parada respiratória com o uso da anestesia peribulbar em humanos (Cangiani, 1997).

A injeção do anestésico local, quer seja dentro do cone músculo-membranoso (retrobulbar), em volta dele (periconal) ou em torno do globo ocular (peribulbar), desloca o globo ocular e a pálpebra para frente, caracterizando a proptose (Vanetti, 1996). O deslocamento do globo ocular é um sintoma importante na realização dos bloqueios oftálmicos (Teixeira et al., 1999). No entanto, é importante que a proptose seja reversível, pois ela causa a elevação da pressão intraocular (Katayama et al., 1996).

\footnotetext{
* Professor. Curso de Medicina Veterinária. Universidade Camilo Castelo Branco (Unicastelo). Av. Hilário da Silva Passos 950, Parque Universitário. Descalvado, SP. 13690-000, Brasil. *Autor para correspondência: dantasmota@yahoo.com.br

** Professor. Faculdade de Medicina Veterinária. Universidade Federal de Uberlândia. Uberlândia, MG.

*** Professora. Faculdade de Medicina Veterinária. Universidade Federal do Espírito Santo (UFES).

${ }^{* * * *}$ Acadêmica do Curso de Medicina Veterinária. UNICASTELO. Descalvado-SP.
} 
Vários agentes anestésicos foram empregados nos bloqueios oftálmicos como a lidocaína a $2 \%$ em bovinos (Ramakrishna, 1995) e caninos (Hazra e Samanta, 1998), a bupivacaína a 0,5\% em caninos (Deluca, 1994), bupivacaína 0,75\% associada à lidocaína a $2 \%$ com adrenalina em humanos (Teixeira et al., 1999) e a ropivacaína a 1\% em humanos (Serzedo et al., 2000) e em cães (Costa et al., 2005; Bevilacqua et al., 2006; Klaumann, 2007).

A ropivacaína tem sido utilizada nos bloqueios peribulbares, para a realização de cirurgias intra-oculares em humanos (Serzedo et al. 2001). Este agente pertence ao grupo aminoamidas, possui efeito vasoconstritor que influencia no volume sanguíneo local ocasionando diminuinção da pressão intraocular (Serzedo et al., 2000).

Sua ação é restrita ao local de aplicação e reverte-se rapidamente quando utilizada em formulações menos concentradas que, aliado a sua baixa toxicidade sistêmica, fazem da ropivacaina uma opção interessante para a realização de bloqueios nervosos, tanto cen-trais quanto periféricos (Lemke e Dawson, 2000; Massone, 2002).

O objetivo deste trabalho foi verificar os efeitos da ropivacaína a $0,5 \%$ na anestesia peribulbar em gatos.

\section{Material e métodos}

Foram utilizados 10 gatos sem raça definida adultos, machos e fêmeas, com peso entre 2 a $3 \mathrm{~kg}$. Após jejum de seis horas, de alimentos sólido e líquido, administrou-se como medicação pré-anestésica a clorpromazina (1,0 mg/kg VO). Transcor-ridos 20 minutos promoveu-se indução com propofol (3,0 mg/kg EV), apenas para facilitar a realização do bloqueio anestésico. Foi feito anti-sepsia local no olho direito com polivinil-pirrolidona em solução fisiológica a 0,9\%, 1:50. Após 10 minutos da administração do propofol, foi realizado, no olho direito, bloqueio peribulbar com ropivacaína a $0,5 \%$ (1,0 mg/kg), com seringa de $1,0 \mathrm{~mL}$ e agulha hipodérmica de 13 x 3,8 mm em forma de "L". O anestésico foi administrado na porção superior da cavidade orbitária em direção ao fórnice na posição súpero-mediana (Figura 1- $A, B$ e $C$ ).

Após a aplicação do volume total da ropivacaína, avaliou-se: início da proptose; regressão da proptose; presença de nistagmo; início da perda do reflexo corneano; duração da ausência do reflexo corneano; início da midríase; duração da midríase; início da perda do reflexo óculo-cefálico; duração da ausência do reflexo óculo-cefálico, utilizando como controle o olho esquerdo, do mesmo animal, que não sofreu bloqueio anestésico (Tabela 1).

Foram aferidas as frequências cardíaca (FC) e respiratória (FR) nos momentos anteriores à administração da clorpromazina, 20 minutos após administração da clorpromazina (T0), 20 minutos após o bloqueio peribulbar com a ropivacaina a $0,5 \%$ (T20) e a cada 20 minutos até a volta da sensibilidade na região anestesiada (Tabela 2 ).
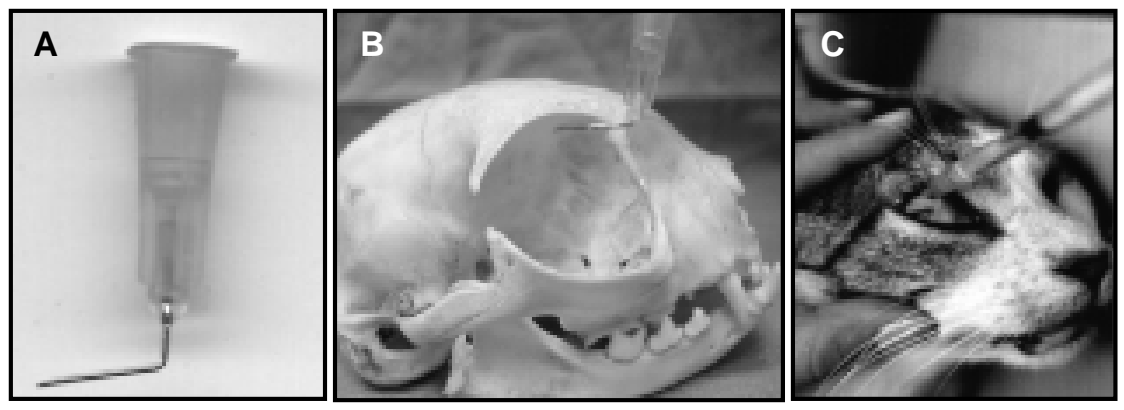

Figura 1: Formato em "L" da agulha hipodérmica utilizada para o bloqueio peribulbar em gatos com ropivacaína $(A)$. Nota-se a posição da agulha na porção superior mediana da cavidade orbitária em direção ao fórnice (B) e local de aplicação do anestésico (C).

Tabela 1: Valores médios ${ }^{1}(n=10)$ com seus respectivos desvios-padrão e análise de significância estatística para a observação no tempo da ocorrência de midríase, proptose, perda do reflexo óculo-cefálico e corneano em gato submetidos a administração peribulbar de ropivacaína 0,5\%

\begin{tabular}{l|cccc}
\hline \multirow{2}{*}{ Observação no Tempo } & \multicolumn{4}{|c}{ Parâmetro analisado (Minutos após a administração peribulbar) } \\
\cline { 2 - 5 } & MID $^{2}$ & PPT $^{2}$ & ROC $^{2}$ & PRC $^{2}$ \\
\hline Início do efeito & $10,60 \pm 1,65^{\mathrm{A}}$ & $10,10 \pm 1,52^{\mathrm{A}}$ & $11,20 \pm 1,99^{\mathrm{A}}$ & $10,50 \pm 2,32^{\mathrm{A}}$ \\
Término do efeito & $94,00 \pm 32,47^{\mathrm{B}}$ & $110,70 \pm 22,17^{\mathrm{B}}$ & $132,70 \pm 37,03^{\mathrm{B}}$ & $134,70 \pm 37,46^{\mathrm{B}}$ \\
Duração do efeito & $83,40 \pm 32,48^{\mathrm{B}}$ & $100,60 \pm 21,85^{\mathrm{B}}$ & $121,50 \pm 36,75^{\mathrm{B}}$ & $124,20 \pm 37,14^{\mathrm{B}}$ \\
\hline Valor de F & 65,8 & 109,52 & 204,91 & 107,32 \\
Pr>F & $<0,0001$ & $<0,0001$ & $<0,0001$ & $<0,0001$ \\
\hline
\end{tabular}

${ }^{1}$ Médias seguidas pela mesma letra, maiúscula na coluna, não diferem entre si pelo teste $T(P e " 0,05)$.

${ }^{2}$ MID = Midríase; PPT = Proptose; ROC = Perda do reflexo óculo-cefálico; PRC = Perda do reflexo corneano.

${ }^{3}$ Probabilidade de significância do valor $\mathrm{F}$. 
Tabela 2: Valores médios ${ }^{1}(n=10)$ com respectivos desviospadrão e análise de significância estatística da frequência respiratória e cardíaca, aferidas em gatos sobre efeitos da anestesia peribulbar com ropivacaína $0,5 \%$

\begin{tabular}{ccc}
\hline Momentos $^{2}$ & $\begin{array}{c}\text { Frequência } \\
\text { Respiratória } \\
(\mathrm{mpm})\end{array}$ & $\begin{array}{c}\text { Frequência } \\
\text { Cardíaca } \\
(\mathrm{bpm})\end{array}$ \\
\hline T0 & $46,2 \pm 15,8^{\mathrm{A}}$ & $163,6 \pm 31,0^{\mathrm{A}}$ \\
T20 & $43,9 \pm 10,0^{\mathrm{AB}}$ & $181,3 \pm 28,5^{\mathrm{A}}$ \\
T40 & $43,2 \pm 8,0^{\mathrm{AB}}$ & $192,6 \pm 23,5^{\mathrm{A}}$ \\
T60 & $40,7 \pm 7,4^{\mathrm{AB}}$ & $190,7 \pm 22,7^{\mathrm{A}}$ \\
T80 & $37,8 \pm 10,9^{\mathrm{AB}}$ & $186,4 \pm 26,0^{\mathrm{A}}$ \\
T100 & $36,1 \pm 9,7^{\mathrm{AB}}$ & $187,1 \pm 15,8^{\mathrm{A}}$ \\
T120 & $38,1 \pm 6,4^{\mathrm{AB}}$ & $188,5 \pm 16,0^{\mathrm{A}}$ \\
T140 & $39,0 \pm 7,2^{\mathrm{AB}}$ & $187,3 \pm 22,7^{\mathrm{A}}$ \\
T160 & $36,6 \pm 5,9^{\mathrm{AB}}$ & $195,2 \pm 25,1^{\mathrm{A}}$ \\
T180 & $37,3 \pm 3,8^{\mathrm{AB}}$ & $186,7 \pm 30,6^{\mathrm{A}}$ \\
T200 & $27,0 \pm 3,0^{\mathrm{B}}$ & $204,0 \pm 50,9^{\mathrm{A}}$ \\
\hline Valor de F & 2,73 & 1,12 \\
Pr> $^{3}$ & $\mathbf{0 , 0 0 5 2}$ & $\mathbf{0 , 3 5 8 5}$ \\
\hline Medis
\end{tabular}

${ }^{1}$ Médias seguidas pela mesma letra, maiúscula na coluna, não diferem entre si pelo teste $\mathrm{T}(\mathrm{Pe}=0,05)$

2 Tempo de aferição das frequências respiratória e cardíaca, após administração da ropivacaína $0,5 \%$, via peribulbar.

${ }^{3}$ Probabilidade de significância do valor F.

mpm: movimentos por minuto.

bpm: batimentos por minuto.

Os dados obtidos foram submetidos a análise de significância estatística em um delineamento inteiramente casualizado e a comparação das médias pelo Teste T-Student Pareado (Pe ${ }^{\text {TM5) }}$.

\section{Resultados e discussão}

A sedação é necessária para realização dos bloqueios anestésicos em oftalmologia (Cangiani, 1997). Neste trabalho, a utilização do pré-anestésico clorpromazina e do anestésico propofol facilitou o bloqueio peribulbar em gatos. O protocolo anestésico foi utilizado por possuir um período hábil de 1015 minutos (Massone 2003) e por não prejudicar a avaliação dos parâmetros oculares.

Muir III et al. (2001) recomendaram a dosagem de ropivacaína para cães e gatos de 1,0-3,0 mg/kg para bloqueios nervosos seletivos. Neste trabalho optou-se pela dose de $1,0 \mathrm{mg} / \mathrm{kg}$, sendo a mesma dose preconizada por Milken et al. (2006) no bloqueio do nervo alveolar mandibular em gatos, observando efetividade no bloqueio peribulbar, sem sinal aparente de toxicidade.

Nesta presente pesquisa, com o uso da ropivacaína a $0,5 \%$ no bloqueio peribulbar em gatos não foi observada reação adversa como prurido, blefaroedema, quemose e blefaroespasmo, conforme relatos de Klaumann (2007), ao realizarem o bloqueio peribulbar em cães com ropivacaína a 1\%. A este resultado atribuiu-se à concentração do anestésico aqui utilizado de $0,5 \%$, o que permitiu também o uso de um volume satisfatório do anestésico, evitando desta forma falha no bloqueio (Milken et al., 2006).
Ramakrishna (1995) verificou em três bovinos, após o uso de lidocaína retrobulbar, apnéia respiratória devido à injeção do anestésico no espaço sub-aracnóide, junto à bainha do nervo óptico, resultante da dispersão do fármaco para o sistema nervoso central. Tais efeitos colaterais não foram observados neste estudo, provavelmente por ter sido usada para a aplicação do anestésico uma agulha encurvada de calibre pequeno de $13 \times 3,8 \mathrm{~mm}$ e por ter-se escolhido a técnica peribulbar, que apresenta risco quase nulo, de injuria ao nervo óptico, pois a agulha não penetra no cone muscular, e o risco de injeção intravenoso acidental do anestésico local também fica bastante reduzido (Ripart et al., 2001; Cangiani, 2005).

A instalação da proptose ocorreu em média após 10 minutos e um segundo da aplicação do anestésico, evidenciando a eficácia na técnica aqui utilizada. Se a injeção do anestésico local for feita inadvertidamente através da bainha do nervo óptico, não causando protusão do globo ocular, o agente passará para o líquido cefalorraquidiano ou para o olho adelfo, podendo causar parada cardiorrespiratoria (Texeira et al., 1999).

O período observado para a regressão da proptose foi em média de 100 minutos e seis segundos. De acordo com Teixeira et al. (1999), a ausência na regressão da protusão do globo ocular esta relacionada com o aumento da pressão intraocular.

A avaliação da sensibilidade tátil da córnea é necessária para avaliação dos efeitos do bloqueio anestésico (Lucci et al., 2004). A ausência do reflexo corneano ocorre devido ao bloqueio dos ramos do nervo trigêmio e abducente (Mazzanti et al., 1999). Verificou-se que a perda do reflexo corneano ocorreu em média após 10 minutos e cinco segundos do bloqueio peribulbar em gatos. Observações coincidentes foram notadas por Serzedo et al. (2001) ao utilizarem ropivacaína a $1 \%$ e bupivacaína a $0,75 \%$, na anestesia peribulbar de humanos. Com a ropivacaína a 0,5\% notou-se ausência do reflexo corneano de 124 minutos e dois segundos em média. Entretanto, Deluca (1994) no bloqueio peribulbar com lidocaína em humanos, ressaltou ausência de reflexo corneano por 17 minutos e trinta e sete segundos. A boa durabilidade da ropivacaína é devida à sua longa duração e por apresentar alta afinidade por proteínas plasmáticas, sendo três a quatro vezes mais potentes que a lidocaína (Olmez et al., 2004).

A midríase com duração de 83 minutos em média, observada neste estudo, foi devida ao bloqueio do nervo oculomotor, afetando assim a inervação parassimpática do esfíncter pupilar, causando sua dilatação (Mazzanti et al., 1999). Tratase de um fator importante para realizações de procedimentos cirúrgicos intraoculares, sendo desejável a midríase completa (Carareto et al., 2006).

A acinesia, imobilidade completa do bulbo ocular é de grande valor para o acesso do espaço intraocular, pois em planos anestésicos cirúrgico, o bulbo ocular de animais de pequeno porte rotaciona medial e ventralmente na órbita e ocorre exposição da terceira pálpebra, escondendo total ou parcialmente a córnea, sendo nestes casos comum o uso de bloqueadores neuromusculares para a centralização do bulbo. Outros fármacos e técnicas podem produzir efeito semelhante, com maior segurança para o paciente (Bechara 
2002; Carareto et al., 2006), como a ropivacaína em bloqueios peribulbares, que apresenta boa eficácia e segurança nas técnicas cirúrgicas de catarata, além de produzir 12 horas de analgesia pós-operatória (Lew et al., 2001; Martini et al., 2002; Olmez et al., 2004).

$\mathrm{O}$ aparelho vestibular transmite informações para os nervos cranianos oculomotor (III), troclear (IV) e abducente (VI), responsáveis pela inervação da musculatura extrínseca do bulbo ocular. No movimento giratório da cabeça de um cão, no sentido horário ou anti-horário, ocorre nistagmo lateral, conhecido como reflexo oculocefálico. No bloqueio anestésico dos nervos III, IV e VI, observa-se ausência da atividade da musculatura extrínseca do olho e do reflexo oculocefálico, promovendo acinesia do bulbo ocular (Montiani-Ferreira e Petersen-Jones, 2002; Magalhães et al., 2004). Observações verificadas neste experimento, ao realizar a anestesia peribulbar com ropivacaína a 0,5\%, promovendo ausência do reflexo oculocefálico durante 121 minutos e cinco segundos em média. Período de tempo possivelmente compatível para realização de procedimentos cirúrgicos oftálmicos.

A coordenação do nistagmo é realizada pelo fascículo longitudinal medial, que auxilia na coordenação dos músculos extraoculares (Petersen-Jones e Crispin, 1999). Como a anestesia peribulbar bloqueia os ramos dos nervos oculomotor, troclear e abducente, responsáveis pela inervação dos músculos extraoculares (Mazzanti et al., 1999), ocorre ausência de movimento rápido e involuntário de globo ocular. Episódio verificado em todos os animais, neste experimento, submetidos ao bloqueio do peribulbar com ropivacaína a $0,5 \%$.

Notou-se que não ocorreu perda do reflexo motor palpebral nos gatos após anestesia peribulbar com a ropivacaína. Fato devido à ausência da ação anestésica sobre nervo auriculopalpebral, responsável pela movimentação das pálpebras

\section{Referências}

BECHARA, J.N. Anestesia em oftalmologia. In: FANTONI, D.T.; CORTOPASSI S.R.G. (Ed). Anestesia em cães e gatos. São Paulo: Roca, 2002. p. 271-279.

BERNIS, W.O.; LAZZERI, L. Anestesia geral do cão pelo pentobarbital sódico; observações sobre a dose e o comportamento do pulso arterial, da temperatura e dos movimentos respiratórios. Arq. Esc Sup Vet. Belo Horizonte, v. 10, p. 91-110, 1957.

BEVILACQUA, L.; OLIVA, V.N.L.S.; ANDRADE, A.L.; MATSUBARA L.M.; PERRI S.H.V. Peribulbar anesthesia with ropivacaine as an alternative to neuromuscular blocking agents for cataract surgery in dogs. In 9th World Congress of Veterinary Anaesthesiology, 2006, Santos, SP, p. 192, CBCAV, 2006.

CANGIANI, L.M. Anestesia em oftalmologia. In: MANICA, J. (Ed). Anestesiologia: princípios e técnicas. Artes Médicas: Porto Alegre, 1997, p. 608-615.

CANGIANI, L.M. Retrobulbar ou peribulbar: uma questão de nomenclatura? Rev. Bras. Anest., v. 55, n. 3, p. 134-138, 2005.

CANGIANI, L.M.; OLIVEIRA, A.C.; CAMARGO, L.V.;PEREIRA, A.M.S.A. Sedação com midazolan ou com associação midazolan-fentanil em cirurgia oftálmica sob bloqueio retrobulbar. Rev. Bras. Anest., v. 45, n. 6, p. 363-368, 1995.

CARARETO, R., NUNES, N., FERRO, P.C., NISSHIMORI, C.T., PAULA, D.P., CONCEICAO, E.D.V. Anestesia para Cirurgias Oftalmicas. Rev. Cient. Méd. Vet. v. 4, n. 13, p. 192-202, 2006.
(Mazzanti et al., 1999), visto que no bloqueio peribulbar o anestésico não abrange o nervo facial, que é o principal responsável por esta ação (Cangiani, 1997). Resultado divergente dos encontrados por Chaves et al. (1997) ao realizarem o bloqueio peribulbar em cães com lidocaína $2 \%$.

A diminuição na frequência cardíaca no momento inicial do estudo atribui-se a ação da clorpromazina, que além de causar depressão do sistema reticular, bloqueia no sistema nervoso central e neurotransmissores como a serotonina e dopamina, levando a diminuição da frequência cardíaca (Muir III et al. 2001; Cortopassi e Fantoni 2002).

A mesma avaliação foi realizada para a frequência respiratória, comparando os diversos momentos com T0. Foi observada diferença significativa em relação aos momentos T0 e T200, com redução gradativa. Esses resultados se devem, provavelmente, ao efeito da clorpromazina, que reduz a frequência respiratória por diminuir o metabolismo basal (Bernis e Larezzi, 1957) e do propofol sobre o centro respiratório e no relaxamento dos músculos diafragma e intercostal (Souza et al., 2002).

Apesar das pequenas alterações observadas na frequência cardíaca e respiratória durante o período de avaliação, as mesmas mantiveram próximas da normalidade para esta espécie em descanso (Feitosa, 2004), o que demonstra a eficiência e segurança do procedimento utilizado.

\section{Conclusão}

A ropivacaína a $0,5 \%$ na dose de $1 \mathrm{mg} / \mathrm{kg}$ mostrou-se eficiente na anestesia peribulbar de gatos sem raça definida, não apresentando sinais aparentes de toxicidade. No entanto, esta técnica isoladamente não abole os reflexos palpebrais, devendo ser associada a outros protocolos anestésicos para realização de procedimentos oftálmicos.

CHAVES, N.S.T.; BARROS, P.S.M.; MARTINS, A.F.; ARAÚJO, E.G.; JACOMINI, C.Z. Anestesia peribulbar em cães. Clín. Vet. v. 2, n. 7, p. 12-14, 1997.

COSTA, G.; MUSIC, M.; SPADOLA, F.; SIRACUSANO, L.; CUCINOTTA, G. Comparison of intravenous and peribulbar Cis-Atracurium administration in dogs in undergoing ophthalmic surgery. Vet. Anaest. Analg. v. 32, p. 1-5, 2005.

CORTOPASSI, S.R.G.; FANTONI, D.T. Medicação pré-anestésica. In: FANTONI D.T.; CORTOPASSI S.R.G. (Ed). Anestesia em cães e gatos. São Paulo: Roca, 2002. p. 152-158.

DELUCA, C.A. Anestesia peribulbar em cirurgia oftalmologica. Rev. Arg. Anest. v. 52, n. 1, p. 15-43, 1994.

FEITOSA, F.L.F. Exame físico geral ou de rotina. In: FEITOSA, F.L.F. (Ed). Semiologia Veterinária, a arte do diagnóstico. São Paulo: Roca, 2004. p. 77-102.

FREITAS, A.C.H.; ESPIRANDELLI L.C. Parada respiratória peribulbar: relato de caso. Rev. Brás. Anest, v. 47, n, 2, p. 34-137, 1997.

GELATT, K.N. Veterinary Ophtalmology. Pensilvania: Lea e Febiger, 1991, p. 195-200.

HAZRA, S.; SAMANTA, P.K. Extracapsular extration of canine lens by aspiration technique. Ind. J. Animal. Sci., v. 37, n. 2, p. 29-31, 1998.

KATAYAMA, M., ZAMBOTTI, H.C.; VIEIRA, J.L. Queda da pálpebra como fator determinante do volume da solução de anestésico local no bloqueio peribulbar. Rev. Bras. Anest. v. 46, n. 5, p. 343-350, 1996. 
KLAUMANN, P.R. Bloqueio peribulbar com ropivacaína a $1 \%$ em cães. 2007. 60 f. Tese (Mestrado em Ciências Veterinárias)-Faculdade de Medicina Veterinária, Universidade Federal do Paraná, Curitiba. 2007.

LEMKE, K.A.; DAWSON, S.D. Management of Pain: Local and regional anesthesia. Vet. Clin. North Am. Small Animal. Pract. v. 30, n. 4, p. 839-857, 2000.

LEW, E; VLODKA, J.D; HADZIC, A. Ropivacaine for peripheral nerve blocks: are there advantages? Tech Reg Anesth Pain Manag. v. 5, n. 2, p. 56-59, 2001.

LUCCI, L.M.D.; ITAMI, C.N.; ALVES, R.F.; MONTESANO, F.T.; OSAKI, M.H.; SANT'ANNA, A.E.B.P.P. Efeito do uso da lente escleral cosmética na sensibilidade tátil corneal em phthisis bulbi. Arq. Brás. Oftalm, v. 67, n. 5, p. 733-736, 2004.

MAGALHÃES, E.; GOVÊIA, C.S.; OLIVEIRA, K.B. Bupivacaína racêmica, levobupivacaína e ropicacaína em anestesia loco-regional para oftalmologia: um estudo comparativo. Rev. Assoc. Méd. Bras. v. 50, n. 2, p. 195-198, 2004.

MASSONE, F. Anestesias gerais barbitúricas e não barbitúricas. In: MASSONE, F. (Ed) Anestesiologia Veterinária: farmacologia e técnicas. Rio de Janeiro: Guanabara Koogan, 2003, p. 56-65.

MASSONE, F. Anestesia Local. In: FANTONI, D.T.; CORTOPASSI, S.R. (Ed). Anestesia em cães e gatos. São Paulo: Roca, 2002, p.193-198.

MARTINI, E.; CAVALLINI, G.M.; CAMPI, L.; LUGLI, N.; NERI, G.; MOLINARI, P. Lidocaine versus robivacaine for topical anesthesia in cataract surgery. J. Cataract.Refract.Surg. v. 28, p. 1018-1022, 2002. MAZZANTI, A.; RAISER, A.G.; PIPPI, N.L.; OLIVEIRA, J.C.D.; EURIDES, D.; ANDRADE, C.M. Nervos oculares dos animais domésticos, comparativos e aplicações clínicas. Vet. Not. v. 5, n. 1, p. 119-130, 1999.

MILKEN, V.M.F.; FREITAS, P.M.C.; EURIDES, D.; SILVA, F.C.O., MOTA, F.C.D., ARAUJO, E., REZENDE, R.J., PRIETO, L.A., MELO, M.S., GOULART, M.R. Bloqueio do nervo alveolar mandibular com ropivacaína a 0,5\% em gatos. Ciência Rural, v. 36, n. 2, p. 550-554, 2006.

MONTIANI-FERREIRA, F.; PETERSEN-JONES, S. Neuro-ophthalmology, In: PETERSEN-JONES S. E CRISPIN S. (Ed). Manual of Small Animal Ophthalmology. 2. ed. London: BSAVA, 2002. p. 257-275.
MORGAN, C.M.; SCHATZ, H.; VINE, A.K. Ocular complications associated with retrobulbar block. Ophthalmology, v. 93, p. 14761478. 1988.

MUIR, III W.W.; HUBBELL J.A.E.; SKARDA R.T.; BEDNARSKI R.M. (Ed.). Fármacos e técnicas de anestesia local. In: Manual de Anestesia Veterinária. 3.ed. São Paulo: Artmed, 2001. p. 45-56.

OLMEZ, G.; CAKMAK, S.S.; CACA, I.; UNLU, M.K. Intraocular pressure and quality of blockade in peribulbar anesthesia using ropivacaine or lidocaine with adrenaline: A double-blind randomized study. Tohoku J. Exp. Med. v. 204, p. 203-208, 2004.

PETERSEN-JONES, S.M.; CRISPIN, S.M. (ed). Neuroftalmologia. In: Manual de Oftalmologia en pequeños animales. Madrid: Harcourt Brace, 1999. p. 275-290.

RAMAKRISHNA, O. Following retrobulbar block in cattle. India. Vet. J. v. 72, p. 97-98, 1995.

RIPART, J.; LEFRANT, J.Y.; GOUSSAYE, J.E.; PRAT-PRADAL, D.; VIVIEN, B.; ELEDJAM, J.J. Peribulbar Versus Retrobulbar Anesthesia for Ophthalmic Surgery: An Anatomical Comparsion of Extraconal and Intraconal Injections. Anesthesiology, v. 54, p. 56-52, 2001.

SERZEDO, P.S.M.; NOCITI, J.R.; ZUCCOLOTTO, E.B.; CAGNOLATI, C.A.; FERREIRA, S.B. Pressão intraocular durante bloqueio peribulbar com ropivacaína a 1\%. Rev. Brás. Anest. v. 50, n. 3, p. 251-253, 2000.

SERZEDO, P.S.M., NOCITI, J.R., ZUCCOLOTTO, E.B., CAGNOLATI, C.A., FERRERA, S.B. Intraocular Pressure and Ropivacaine in Peribulbar Block: A Comparative Study with Bupivacaine. Acta Anaest. Scan. v. 45, n. 5, p. 600-602, 2001.

SOUZA, A.P., POMPERMAYER, L.G., LAVOR, M.S.L., DUARTE, T.S., SILVA, R.M.N. Butorfanol na anestesia com propofol em gatas prétratadas com levomepromazina. Ciência Rural, v. 32, n. 4, p. 589594, 2002.

TEIXEIRA, J.J.M.S.; VANETTI, L.F.A.; CANGIANI, L.M.; CAMARGO, F.B.; FERREIRA, A.A. Proptose: um sinal útil para a realização dos bloqueios retrobulbar e peribulbar. Rev. Bras. Anest, v. 49, n. 1, p. 14-18, 1999.

VANETTI, L.F.A. Anestesia para oftalmologia. In: ORTENZI, A.V., TARDELLI, M.A. (Ed). Anestesiologia. Brasil: SAESP, 1996, p. 591606. 